\title{
SPENDENWERBUNG
}

\section{Storytelling im Fundraising}

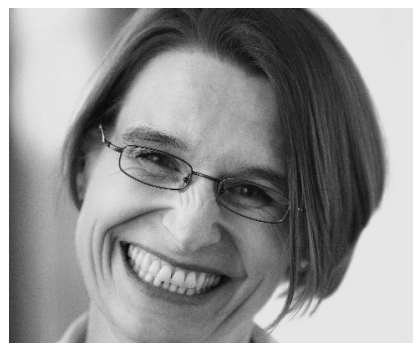

VON ALEXANDRA RIPKEN Alexandra Ripken ist Beraterin und Expertin für Empfehlungsfundraising und Storytelling bei der Agentur "Ziel \& Plan«. Seit 2006 unterstützt sie Stiftungen, Unternehmen und Organisationen in ihrem Sozialmarketing. Internet http://www.blog-adlerauge.de Internet http://www.zielundplan.com

\author{
Wer Spenden bekommen will, muss die Menschen für \\ die Probleme anderer und für die Arbeit sozialer \\ Organisationen interessieren. Am besten gelingt das, \\ wenn man eine gute Geschichte zu erzählen hat.
}

Die Herausforderung von heute ist nicht, ob wir mit den 40- bis 50-Jährigen per E-Mail oder Direct Mail kommunizieren, sondern wie wir sie als Organisation überhaupt erreichen und einbinden, meint Vinay Bhagat von der amerikanische Fundraising-Agentur Convio in seiner Studie » The next generation of American giving «. Darin stellt er fest, dass die Mitglieder dieser »Generation $\mathrm{X}$ « klar werteorientiert spenden. Sie geben dorthin, wohin ihre Altersgenossen bereits geben, und reagieren entsprechend stark auf persönliche Empfehlung.

Doch was empfehlen wir uns gegenseitig? Wir teilen Geschichten miteinander, antwortet der Unternehmensberater und Storyteller Andy Goodman. Informelle Geschichten helfen uns seit Jahrtausenden, in allen Kontinenten und Kulturen, unsere Identität zu begründen. Wir brauchen Geschichten, um unser Leben zu verstehen und zu erklären. Erst Geschichten öffnen uns für die notwendigen Daten.

Geschichten, die wir hören wollen, erzählen von Protagonisten, die keine Opfer sind und unser Mitleid wollen, sondern berichten von Handelnden, die uns über ihre Geschichte schildern, wie wir unser Leben besser bewältigen können. Diese Handelnden sind »Alltagshelden « und damit mehr als Vorbilder.

Held zu sein bedeutet nach dem Marketingexperten Werner Fuchs, Probehandlungen für uns Zuschauer durchzuführen und damit Sinnlücken zu schließen und unser Bedürfnis nach
Sicherheit zu befriedigen. Der Held steht mit seiner Handlung quer zu Verzweiflung und Schicksal. Er überwindet Angst und begründet Hoffnung, die auf Dritte übertragen werden kann. Der Held gibt uns Vertrauen ins Leben, weil er das Leben liebt. Er zeichnet einen Weg. Deshalb hören wir zu.

Vor welchen Aufgaben steht der Held einer Non-Profit-Organisation? Angst zu reduzieren, ist im Kern die entscheidende Aufgabe jeder gemeinnützigen Organisation. Das verdeutlichen bereits wenige Beispiele: Angst vor Einsamkeit - Caritas, Angst vor Arbeitslosigkeit - Obdachlosenhilfe, Angst vor Katastrophen - Rotes Kreuz, Angst vor Umweltzerstörung - Greenpeace, Angst vor Identitätsverlust - Kulturstiftung, Angst vor Chancenlosigkeit Schulstiftung, Angst vor Missbrauch Medica Mondiale. Non-Profit-Organisationen zeigen hier echte Lösungswege auf. Die Hauptperson der Geschichte geht diesen Weg.

Was macht eine gute Geschichte aus? Sie zeigt einen persönlichen Reifungsprozess und vermittelt eine Erkenntnis, die unsere Lebens- und Alltagskompetenz erhöht, sagt der Drehbuchautor und Storyteller Brian McDonald. Reifungsprozess heißt für ihn, durch eine persönliche Hölle zu gehen und danach anders wieder aufzuerstehen.

Inhalt der Story ist ein verbindendes menschliches Gefühl wie Sehnsucht nach Freunden, Hunger nach Liebe, Traum von Frieden oder Wunsch nach gelingendem Leben. Dieses Gefühl ist 
der Corpus der Geschichte, ihre Seele. Als Seele bleibt der Corpus stets verborgen. Die mitgeteilte Erkenntnis zur Erfüllung einer dieser Sehnsüchte ist der Mehrwert für den Empfänger. Beim Gang durch die Hölle hilft die NonProfit-Organisationen dem Einzelnen. Sie mildert seinen Schmerz. Für diesen spezifischen Mehrwert steht die Organisation, dafür bekommt sie Spenden. Brian McDonald empfiehlt ihnen, die Geschichte knapp wie ein Märchen zu erzählen:

Satz 1: Es war einmal: Um wen geht es wo und wann?

Satz 2: Und jeden Tag: Wie lebt der Protagonist tagein, tagaus?

Satz 3: Bis eines Tages: Welches Ereignis wirbelt alles durcheinander?

Satz 4: Und deswegen: Der Held wacht auf, welche Lösung findet er?
Satz 5: Und deswegen: Falls ja, welche zweite Hürde muss er nehmen?

Satz 6: Bis endlich: Wie kehrt die Schlüsselfigur zurück in seinen Alltag?

Satz 7: Und seitdem: Was sagt die Geschichte dem Helden und uns Zuschauern?

Schildern Sie solche Storys realer Menschen in Ihren Direct Mails, im persönlichen Gespräch mit Großspendern oder Unternehmern, präsentieren Sie sie auf Ihrer Website in Wort und Bild oder als Clip auf You Tube, wie die MS Society ihren Film » Beautiful Day «.

So schaffen Sie Gesprächsanlässe und kommen erfolgreich ins Gespräch mit den 40- bis 50-Jährigen.

Untersuchung Convio, Internet http://www. convio.com/files/next-gen-whitepaper.pdf. Video MS Society, Internet http://www.youtube.com/watch?v=tHZ3dwljzcM

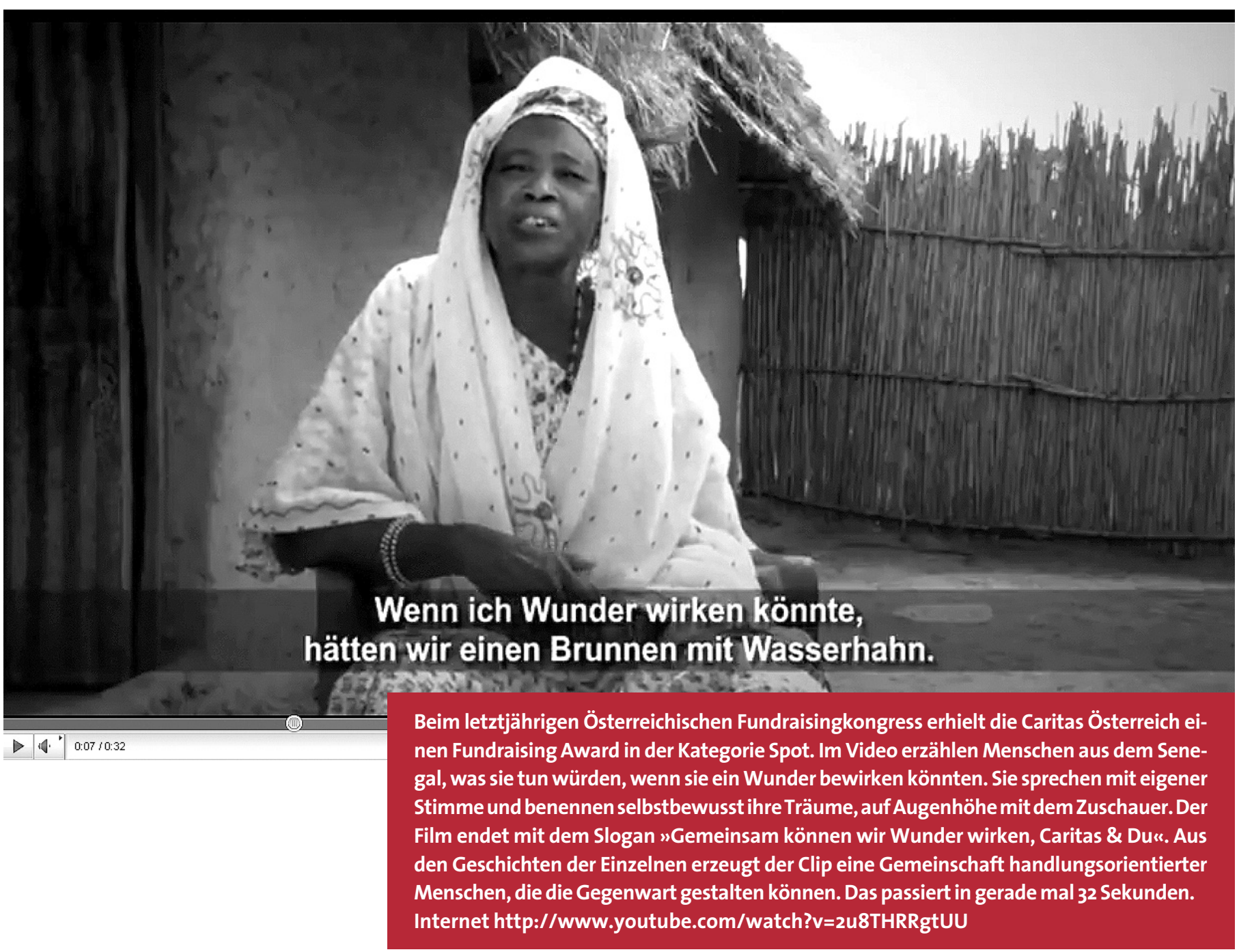

